Research Article

\title{
Study of Two Kinds of Quasi AG-Neutrosophic Extended Triplet Loops
}

\author{
Xiaogang An $\left.{ }^{1}\right)^{1}$ and Mingming Chen $\mathbb{D D}^{1,2}$ \\ ${ }^{1}$ School of Arts and Sciences, Shaanxi University of Science \& Technology, Xi'an 710021, China \\ ${ }^{2}$ Shaanxi Joint Laboratory of Artificial Intelligence, Shaanxi University of Science \& Technology, Xi'an 710021, China
}

Correspondence should be addressed to Xiaogang An; anxiaogang@sust.edu.cn

Received 27 December 2020; Revised 27 January 2021; Accepted 18 February 2021; Published 8 March 2021

Academic Editor: Parimala Mani

Copyright (C) 2021 Xiaogang An and Mingming Chen. This is an open access article distributed under the Creative Commons Attribution License, which permits unrestricted use, distribution, and reproduction in any medium, provided the original work is properly cited.

\begin{abstract}
Abel-Grassmann's groupoid and neutrosophic extended triplet loop are two important algebraic structures that describe two kinds of generalized symmetries. In this paper, we investigate quasi AG-neutrosophic extended triplet loop, which is a fusion structure of the two kinds of algebraic structures mentioned above. We propose new notions of AG-(l,r)-Loop and AG-( $r$, $l)$-Loop, deeply study their basic properties and structural characteristics, and prove strictly the following statements: (1) each strong AG$(l, r)$-Loop can be represented as the union of its disjoint sub-AG-groups, (2) the concepts of strong AG- $(l, r)$-Loop, strong AG-(l,l)Loop, and AG-(l,lr)-Loop are equivalent, and (3) the concepts of strong AG- $(r, l)$-Loop and strong AG- $(r, r)$-Loop are equivalent.
\end{abstract}

\section{Introduction}

The so-called left almost semigroup (LA-semigroup) was actually the concept of an Abel-Grassmann's groupoid (AGgroupoid), which was put forward by Kazim and Naseeruddin [1] at the first time in 1972. Different classes of AGgroupoids and their concerned characteristics have been studied in [2-5].

Neutrosophic set (NS) was first put forward by Smarandache in [6]. Then, it has been growing promptly over the previous 15 years. Nowadays, NS theory is widely used in a couple of sectors such as professional selection [7], integrated speech and text sentiment analysis [8], finite automata [9], clustering methods [10], and deep learning [11]. Besides, more new theoretical studies on NS in [12-17] have been conducted and a few significant results have been gained.

The concept of Abel-Grassmann's neutrosophic extended triplet loop (AG-NET-Loop), which plays a significant role in neutrosophic triplet algebraic structures, was proposed in [18], that is, an AG-NET-Loop is both an AGgroupoid and a neutrosophic extended triplet loop (NETLoop). In [19], the concept of neutrosophic triplet elements (NT-elements) and quasi neutrosophic triplet loops were introduced. In [20], two kinds of quasi AG-NET-Loops (AG-(l,l)-Loop and AG- $(r, r)$-Loop) were proposed and their basic properties were investigated. As a continuation of [20], we propose two other kinds of quasi AG-NET-Loops, which are the AG- $(l, r)$-Loop and the AG- $(r, l)$-Loop. We study their properties and analyze their relationship.

The rest of this paper is arranged as follows. In Section 2, some definitions and properties on quasi AG-NET-Loop are given. Some properties and structures about the AG- $(l, r)$ Loop are discussed in Section 3. The relations among four kinds of quasi AG-NET-Loops are analyzed in Section 4. Some properties about the alternative quasi AG-NET-Loops are discussed in Section 5. Lastly, Section 6 presents the summary and the direction of future efforts.

\section{Preliminaries}

A groupoid $(G, *)$ is called an AG-groupoid if it holds the left invertive law, that is, for all $x, y, z \in G$, $(x * y) * z=(z * y) * x$. In an AG-groupoid $(G, *)$ the medial law holds, for all $x_{1}, x_{2}, x_{3}, x_{4} \in G$, $\left(x_{1} * x_{2}\right) *\left(x_{3} * x_{4}\right)=\left(x_{1} * x_{3}\right) *\left(x_{2} * x_{4}\right)$. An AGgroupoid $(G, *)$ is called locally associative if for all 
$x \in G,(x * x) * x=x *(x * x)$. In an AG-groupoid $(G, *)$, for all $x \in G, k \in Z^{+}, x^{k}$ is defined as follows: $x^{1}=x, x^{2}=$ $x * x, x^{3}=x^{2} * x, x^{4}=x^{3} * x, \ldots, x^{k}=x^{k-1} * x$.

Definition 1 (see [21]). Let $G$ be a nonempty set together with a binary operation * . Then, $G$ is called a neutrosophic extended triplet set if, for all $x \in G$, there exist a neutral of " $x$ " and an opposite of " $x$ " (denoted by neut $(x)$ and anti $(x)$, respectively), such that neut $(x)$, anti $(x) \in G$, and neut $(x) * x=x * \operatorname{neut}(x)=x$, anti $(x) * x=x * \operatorname{anti}(x)=$ neut $(x)$. The triplet $(x, \operatorname{neut}(x)$, anti $(x))$ is called a neutrosophic extended triplet (NET).

Definition 2 (see [18]). An NET set $(G, *)$ is called an NETLoop, if, for all $x, y \in G$, one has $x * y \in G$.

Definition 3 (see [18]). An AG-groupoid $(G, *)$ is called an AG-NET-Loop if it is an NET-Loop.

An AG-NET-Loop $G$ is called a commutative AG-NETLoop if for all $x, y \in G, x * y=y * x$.

Theorem 1 (see [18]). Let $(G, *)$ be an AG-NET-Loop. Then,

(1) For all $x \in G$, neut $(x)$ is unique

(2) For all $x \in G$, $(\text { neut }(x))^{2}=\operatorname{neut}(x)$

Definition 4 (see [2]). AG-groupoid $(G, *)$ is called regular if, for all $a \in G$, there exists $m \in G, a=(a * m) * a$.

Definition 5 (see [20]). Let $(G, *)$ be an AG-groupoid. Then, $G$ is called an AG-(l,l)-Loop if, for all $a \in G$, there exist a local $(l, l)$-neutral element of ' $a$ " and a local $(l, l)$-opposite element of" $a$ " (denoted by $\operatorname{nll}(a)$ and oll (a), respectively), such that $\operatorname{nll}(a) \in G$, oll $(a) \in G$, and $\operatorname{nll}(a) * a=a$ and $\operatorname{oll}(a) * a=\operatorname{nll}(a)$.

Definition 6 (see [20]). Let $(G, *)$ be an AG-groupoid. Then, $G$ is called an AG-(r,r)-Loop if, for all $a \in G$, there exist a local $(r, r)$-neutral element of " $a$ " and a local $(r, r)$ opposite element of " $a$ " (denoted by $\operatorname{nrr}(a)$ and $\operatorname{orr}(a)$, respectively), such that $\operatorname{nrr}(a) \in G \operatorname{orr}(a) \in G$, and $a * \operatorname{nrr}(a)=a$ and $a * \operatorname{orr}(a)=\operatorname{nrr}(a)$.

Definition 7. Let $(G, *)$ be an AG-groupoid. Then, $G$ is called an AG-(l,r)-Loop if, for all $a \in G$, there exist a local $(l, r)$-neutral element of " $a$ " and a local $(l, r)$-opposite element of " $a$ " (denoted by $n l r(a)$ and $\operatorname{olr}(a)$, respectively), such that $n \operatorname{lr}(a) \in G, \operatorname{olr}(a) \in G, \quad$ and $\quad n \operatorname{lr}(a) * a=a \quad$ and $a * \operatorname{olr}(a)=n \operatorname{lr}(a)$

Remark 1. For quasi AG-NET-Loop, we will use the notations such as AG-NET-Loop. If $n l r(a)$ and $\operatorname{olr}(a)$ are not unique, then the set of all local $(l, r)$-neutral elements of" $a$ " and the set of all local $(l, r)$-opposite elements of " $a$ " are denoted by $\{n l r(a)\}$ and $\{$ olr $(a)\}$, respectively.
Definition 8. Let $(G, *)$ be an AG-groupoid. Then, $G$ is called an AG- $(r, l)$-Loop if, for all $a \in G$, there exist a local $(r, l)$-neutral element of " $a$ " and a local $(r, l)$-opposite element of" $a$ " (denoted by $\operatorname{nrl}(a)$ and $\operatorname{orl}(a)$, respectively), such that $\operatorname{nrl}(a) \in G, \operatorname{orl}(a) \in G$, and $a * \operatorname{nrl}(a)=a$ and $\operatorname{orl}(a) * a$ $=\operatorname{nrl}(a)$.

Definition 9. Let $(G, *)$ be an AG-(l,r)-Loop. Then, $G$ is called an AG-(l,lr)-Loop if, for all $a \in G, \operatorname{olr}(a) * a=$ $a * \operatorname{olr}(a)=n \operatorname{lr}(a)$.

Definition 10 (see [22]). An AG-groupoid $G$ with a left identity is called an AG-group if each $a \in G$ has an inverse element $a^{\prime}$.

\section{AG- $(l, r)$-Loop and Strong AG- $(l, r)$-Loop}

Theorem 2. Let $(G, *)$ be a groupoid. Then, $G$ is an $A G-$ $(l, r)$-Loop iff it is a regular AG-groupoid.

Proof. Necessity: if $G$ is an AG-(l,r)-Loop, from Definition 7, for all $a \in G$, there exist $n \operatorname{lr}(a), \operatorname{olr}(a) \in G, n l r(a) * a=a$, and $a * \operatorname{olr}(a)=n l r(a)$. We have $(a * \operatorname{olr}(a)) * a=a$. By Definition $4, G$ is a regular AG-groupoid.

Sufficiency: if $G$ is a regular AG-groupoid, from Definition 4, for all $a \in G$, there exists $m \in G$ and $a=(a * m) * a$. Set $n \operatorname{lr}(a)=a * m$, by Definition $7, G$ is an AG- $(l, r)$-Loop.

Example 1 illustrates that an AG-groupoid may be neither an AG-(l,l)-Loop nor an AG-(l,r)-Loop nor an AG$(r, r)$-Loop nor an AG- $(r, l)$-Loop.

Example 1. Let $G=\{1,2,3,4,5,6,7,8\}$, and the definition of operation * on $G$ is shown in Table 1 . There is no $\operatorname{oll}(2), \operatorname{olr}(2), \operatorname{orr}(2)$, and $\operatorname{orl}(2)$ in G. That is, the element " 2 " in $G$ has no local $(l, l)$-opposite element, no local $(l, r)$ opposite element, no local $(r, r)$-opposite element, and no local $(r, l)$-opposite element. From Definitions $5-8, G$ is neither an AG-(l,l)-Loop nor an AG-(l,r)-Loop nor an AG$(r, r)$-Loop nor an AG- $(r, l)$-Loop.

Example 2 illustrates that an AG- $(l, r)$-Loop may be neither an AG-(l,l)-Loop nor an AG-(r,r)-Loop nor an AG$(r, l)$-Loop.

Example 2. Let $G=\{1,2,3,4,5,6,7\}$, and the definition of operation $*$ on $G$ is shown in Table 2 . From Definition 7, $G$ is an AG-(l,r)-Loop. However, there is no oll (2), $n r r(2)$, and $n r l(2)$ in $G$. From Definitions 5, 6, and 8, $G$ is neither an AG$(l, l)$-Loop nor an AG- $(r, r)$-Loop nor an AG- $(r, l)$-Loop.

Definition 11. An AG- $(l, r)$-Loop $(G, *)$ is called a strong AG-(l,r)-Loop if, for all $a \in G, n l r(a)^{2}=n l r(a)$.

Example 3 illustrates that an AG- $(l, r)$-Loop is not always a strong AG- $(l, r)$-Loop.

Example 3. Let $G=\{1,2,3,4,5,6,7\}$, and the definition of operation $*$ on $G$ is shown in Table 3. From Definition 7, $G$ is an AG-(l,r)-Loop. However, $n l r(2)=3,3 * 3=1$; thus, $G$ is not a strong AG- $(l, r)$-Loop. 
TABle 1: Table of Example 1.

\begin{tabular}{lllllllll}
\hline$*$ & 1 & 2 & 3 & 4 & 5 & 6 & 7 & 8 \\
\hline 1 & 1 & 1 & 1 & 1 & 1 & 1 & 1 & 1 \\
2 & 1 & 1 & 1 & 2 & 1 & 1 & 1 & 1 \\
3 & 1 & 1 & 3 & 1 & 1 & 1 & 1 & 1 \\
4 & 1 & 2 & 1 & 4 & 1 & 1 & 1 & 1 \\
5 & 1 & 1 & 1 & 1 & 5 & 1 & 1 & 1 \\
6 & 1 & 1 & 1 & 1 & 1 & 6 & 8 & 8 \\
7 & 1 & 1 & 1 & 1 & 1 & 8 & 7 & 8 \\
8 & 1 & 1 & 1 & 1 & 1 & 8 & 8 & 8 \\
\hline
\end{tabular}

TABle 2: Table of Example 2.

\begin{tabular}{llllllll}
\hline$*$ & 1 & 2 & 3 & 4 & 5 & 6 & 7 \\
\hline 1 & 1 & 1 & 1 & 1 & 1 & 1 & 1 \\
2 & 1 & 1 & 1 & 4 & 4 & 1 & 1 \\
3 & 1 & 1 & 3 & 1 & 3 & 3 & 7 \\
4 & 1 & 2 & 1 & 1 & 2 & 1 & 1 \\
5 & 1 & 2 & 3 & 4 & 5 & 3 & 7 \\
6 & 1 & 1 & 3 & 1 & 3 & 6 & 7 \\
7 & 1 & 1 & 7 & 1 & 7 & 7 & 7 \\
\hline
\end{tabular}

TABle 3: Table of Example 3.

\begin{tabular}{llllllll}
\hline$*$ & 1 & 2 & 3 & 4 & 5 & 6 & 7 \\
\hline 1 & 1 & 1 & 1 & 1 & 1 & 1 & 1 \\
2 & 1 & 1 & 3 & 3 & 1 & 3 & 3 \\
3 & 1 & 2 & 1 & 2 & 1 & 2 & 2 \\
4 & 1 & 2 & 3 & 4 & 5 & 6 & 7 \\
5 & 1 & 1 & 1 & 5 & 5 & 1 & 1 \\
6 & 1 & 2 & 3 & 6 & 1 & 6 & 6 \\
7 & 1 & 2 & 3 & 7 & 1 & 6 & 7 \\
\hline
\end{tabular}

Example 4 illustrates that a strong AG- $(l, r)$-Loop is not always an AG-NET-Loop.

Example 4. Let $G=\{1,2,3,4,5,6,7\}$, and the definition of operation * on $G$ is shown in Table 4 . By Definition $11, G$ is a strong AG-(l,r)-Loop. However, since $1 * 4 \neq 4 * 1, G$ is not an AG-NET-Loop.

Theorem 3. Let $(G, *)$ be a strong $A G-(l, r)$-Loop. Then,

(1) For all $a \in G, n l r(a)$ is unique

(2) For all $a \in G, n l r(n l r(a))=n l r(a)$

(3) For all $a \in G$ and for any $r \in\{\operatorname{olr}(a)\}, n l r(a) * r \in\{\operatorname{olr}(a)\}$

(4) For all $a, b \in G$, nlr $(a * b)=n l r(a) * n l r(b)$

Proof

(1) If $(G, *)$ is a strong AG- $(l, r)$-Loop, suppose $a \in G$, there exist $n l r_{1}, n l r_{2} \in\{n l r(a)\}$. By Definition 11, $n l r_{1} * a=a, n l r_{2} * a=a, \quad n l r_{1} * n l r_{1}=n l \quad r_{1}$, and
Table 4: Table of Example 4.

\begin{tabular}{llllllll}
\hline$*$ & 1 & 2 & 3 & 4 & 5 & 6 & 7 \\
\hline 1 & 1 & 1 & 3 & 4 & 1 & 1 & 1 \\
2 & 1 & 2 & 3 & 4 & 1 & 1 & 1 \\
3 & 4 & 4 & 1 & 3 & 4 & 4 & 4 \\
4 & 3 & 3 & 4 & 1 & 3 & 3 & 3 \\
5 & 1 & 1 & 3 & 4 & 5 & 1 & 1 \\
6 & 1 & 1 & 3 & 4 & 1 & 6 & 6 \\
7 & 1 & 1 & 3 & 4 & 1 & 6 & 7 \\
\hline
\end{tabular}

$n l r_{2} * n l r_{2}=n l r_{2}$, and there exist $o l r_{1}, o l r_{2} \in G$ which satisfy $a * o l r_{1}=n l r_{1}$ and $a * o l r_{2}=n l r_{2}$. We have

$$
\begin{aligned}
n l r_{1} * n l r_{2}= & \left(n l r_{1} * n l r_{1}\right) * n l r_{2} \\
= & \left(n l r_{2} * n l r_{1}\right) * n l r_{1} \\
= & \left(n l r_{2} * n l r_{1}\right) *\left(a * \text { ol }_{1}\right) \\
= & \left(n l r_{2} * a\right) *\left(n l r_{1} * \text { ol } r_{1}\right) \\
& (\text { by the medial law }) \\
= & \left(n l r_{1} * a\right) *\left(n l r_{1} * \text { ol } r_{1}\right) \\
= & \left(n l r_{1} * n l r_{1}\right) *\left(a * \text { ol } r_{1}\right) \\
& (\text { by the medial law }) \\
= & n l r_{1} * n l r_{1}=n l r_{1}, \\
n l r_{2} * n l r_{1}= & \left(n l r_{2} * n l r_{2}\right) * n l r_{1} \\
= & \left(n l r_{1} * n l r_{2}\right) * n l r_{2} \\
= & \left(n l r_{1} * n l r_{2}\right) *\left(a * \text { ol } r_{2}\right) \\
= & \left(n l r_{1} * a\right) *\left(n l r_{2} * \text { ol } r_{2}\right) \\
& (b y \text { the medial law }) \\
= & \left(n l r_{2} * a\right) *\left(n l r_{2} * \text { ol } r_{2}\right) \\
= & \left(n l r_{2} * n l r_{2}\right) *\left(a * o l r_{2}\right) \\
& (b y \text { the medial law }) \\
= & n l r_{2} * n l r_{2}=n l r_{2}, \\
n l r_{2}= & n l r_{2} * n l r_{1} \\
= & \left(n l r_{2} * n l r_{2}\right) * n l r_{1} \\
= & \left(n l r_{1} * n l r_{2}\right) * n l r_{2} \\
= & n l r_{1} * n l r_{2}=n l r_{1} . \\
&
\end{aligned}
$$

We know that $n l r_{2}=n l r_{1}$, and $n l r(a)$ is unique.

(2) If $(G, *)$ is a strong AG- $(l, r)$-Loop, from Definition 11 , we have, for all $a \in G, n l r(a)^{2}=n l r(a)$. Thus, $n l r(n l r(a))=n l r(a)$.

(3) Suppose $r \in\{\operatorname{olr}(a)\}$; then,

$$
\begin{aligned}
a *(n l r(a) * r) & =(n \operatorname{lr}(a) * a) *(n \ln (a) * r) \\
& =(n \operatorname{lr}(a) * n \operatorname{lr}(a)) *(a * r) \quad(\text { by the medial law }) \\
& =n \operatorname{lr}(a) * n \operatorname{lr}(a) \\
& =n \operatorname{lr}(a) .
\end{aligned}
$$

So, we get $n l r(a) * r \in\{\operatorname{olr}(a)\}$.

(4) From Definition 11, we have, for all $a, b \in G$, 


$$
\begin{aligned}
a * b & =(n l r(a) * a) *(n l r(b) * b) \\
& =(n \operatorname{lr}(a) * n l r(b)) *(a * b) \\
n l r(a) * n l r(b) & =(a * \operatorname{olr}(a)) *(b * \operatorname{olr}(b)) \\
& =(a * b) *(\operatorname{olr}(a) * \operatorname{olr}(b))
\end{aligned}
$$

Therefore, $n l r(a * b)=n l r(a) * n l r(b)$.

Example 5. Let $G=\{1,2,3,4,5,6,7\}$, and the definition of operation $*$ on $G$ is shown in Table 5 . It is a strong AG- $(l, r)$ Loop. We have (corresponding to the results of Theorem 3 )

(1) For all $a \in G$, we can verify that $n \operatorname{lr}(a)$ is unique.

(2) Being $n l r(n l r(1))=n l r(1), n l r(n l r(2))=n l r(2)$, $n l r(n l r(3))=n l r(3), n l r(n l r(4))=n l r(4), n l r(n l r$ (5)) $=n l r(5), n l r(n l r(6))=n l r(6)$, and $n l r(n l r(7))$ $=n l r(7)$, that is, for all $a \in G, n l r(n l r(a))=n l r(a)$.

(3) For any $a \in G$, let $a=1$, and we can get $n l r(1)=1$ and $\{\operatorname{olr}(1)\}=\{1,2,5,6,7\}$. Being $1 * 1=1 * 2=$ $1 * 5=1 * 6=1 * 7=1 \in\{\operatorname{olr}(1)\}$, that is, $n \operatorname{lr}(1) * o$ $\operatorname{lr}(1) \in\{\operatorname{olr}(1)\}$, let $a=3$, and we can get $n \operatorname{lr}(3)=1$, $\operatorname{olr}(3)=3$. Being $1 * 3=3=\operatorname{olr}(3)$, that is, $n \operatorname{lr}(3) * \operatorname{olr}(3) \in\{\operatorname{olr}(3)\}$, we can verify other cases; thus, $n l r(a) * r \in\{\operatorname{olr}(a)\}$.

(4) For any $a, b \in G$, without loss of generality, let $a=1$ and $b=3$; we can get $n l r(1 * 3)=n l r(1) * n l r(3)$. We can verify other cases; thus, $n l r(a * b)=n l r(a) * n l r(b)$.

Theorem 4. Let $(G, *)$ be a strong $A G-(l, r)$-Loop. A binary $\approx$ on $G$ is introduced as follows:

$$
\text { for all } a, b \in G, a \approx b \Leftrightarrow n l r(a)=n l r(b) \text {. }
$$

Then,

(1) The binary $\approx$ on $G$ is an equivalence relation, and the equivalent class contained $x$ is denoted by $[x]_{\approx}$

(2) For all $x \in G,[x]_{\approx}$ is a sub-AG-group

(3) $G=\cup_{x \in G}[x]_{\approx}$, that is, each strong AG-(l,r)-Loop can be represented as the union of its disjoint sub-AGgroups

\section{Proof}

(1) From the binary $\approx$ definition, it is easy to verify that $\approx$ has the properties of reflexive, symmetric, and transitive. Thus, it is an equivalence relation.

(2) For all $a \in[x]_{\approx}$, let $n l r(x)=e_{x}$, and we have $n l r(a)=n l r(x)=e_{x}$. From Theorem 3 (2), $n \operatorname{lr}\left(e_{x}\right)=e_{x}$, and we have $e_{x} \in[x]_{\approx}$ :

(i) By Definition 11, we have $e_{x} * a=n l r(a) * a=a$; thus, $e_{x}$ is a left identity of $[x]_{\approx}$.

(ii) For all $a, b, c \in[x]_{\approx}$, the left invertive law holds directly.
TABle 5: Table of Example 5.

\begin{tabular}{llllllll}
\hline$*$ & 1 & 2 & 3 & 4 & 5 & 6 & 7 \\
\hline 1 & 1 & 1 & 3 & 4 & 1 & 1 & 1 \\
2 & 1 & 2 & 3 & 4 & 1 & 1 & 2 \\
3 & 4 & 4 & 1 & 3 & 4 & 4 & 4 \\
4 & 3 & 3 & 4 & 1 & 3 & 3 & 3 \\
5 & 1 & 1 & 3 & 4 & 5 & 1 & 5 \\
6 & 1 & 1 & 3 & 4 & 1 & 6 & 1 \\
7 & 1 & 2 & 3 & 4 & 5 & 1 & 7 \\
\hline
\end{tabular}

(iii) For all $a, b \in[x]_{\approx}, n l r(a)=n l r(b)=e_{x}$; from Theorem 3 (4), $n l r(a * b)=n l r(a) * n l r(b)=e_{x}$; thus, $a * b \in[x]_{\approx}$.

(iv) For all $a \in[x]_{\approx}$, let $n l r(a)=e_{x}$, and suppose $p \in\{\operatorname{olr}(a)\}, q=n \operatorname{lr}(a) * p$; by Theorem 3 (3), we have $q \in\{\operatorname{olr}(a)\}, a * q=n \operatorname{lr}(a)=e_{x}$, and

$$
\begin{aligned}
n l r(q) & =n l r(n l r(a) * p) \\
& =n l r(n l r(a)) * n l r(p) \quad(\text { by Theorem 3(4)) } \\
& =n l r(a) * n l r(p) \quad(\text { by Theorem 3(2)) } \\
& =n l r(a * p) \quad(\text { by Theorem 3(4)) } \\
& =n l r(n l r(a)) \\
& =n l r(a) \quad(\text { by Theorem 3(2)) } \\
& =e_{x} .
\end{aligned}
$$

(v) $q * a=(n l r(q) * q) * a=\left(e_{x} * q\right) * a=$ $(a * q) * e_{x}=e_{x}$. Thus, $q \in[x]_{\approx}$ and $q$ is an inverse element of $a$. From Definition $10,[x]_{\approx}$ is a sub-AGgroup of $G$.

(3) By Theorem $3(1)$, for all $a \in[x]_{\approx}, n l r(a)$ is unique. Then, $G=\cup_{x \in G}[x]_{\approx}$.

Example 6. Let $G=\{1,2,3,4,5,6,7,8\}$, and the definition of operation $*$ on $G$ is shown in Table $6 .[1]_{\approx}=\{1,2,3,4\}$ and $[5]_{\approx}=\{5,6,7,8\} . G=[1]_{\approx} \cup[5]_{\approx}$, and $[1]_{\approx}$ and $[5]_{\approx}$ are subAG-groups of $G$.

Let $G$ be an AG-groupoid; then, $a$ is an idempotent in $G$ if $a \in G, a^{2}=a$. The set of all idempotents in $G$ is denoted by $E(G)$. An AG-groupoid $G$ is called an AG-band if $G=E(G)$.

From now on, we assume that $G$ is a strong AG- $(l, r)$ Loop, which is the same as Theorem 4. Let $Y$ be an AG-band, $Y \subset G$, and for any $\alpha \in Y$, the equivalent class $[\alpha]_{\approx}$, which is defined in Theorem 4 , will be denoted by $S_{\alpha}$, and the elements of $S_{\alpha}$ will be denoted by $a_{\alpha}, b_{\alpha}, \ldots$, .

Theorem 5. Let $(G, *)$ be a groupoid, $Y$ be an $A G$-band, $Y \subset G . G=\cup_{\alpha \in Y} S_{\alpha},\left(S_{\alpha}, *\right)$ is a strong AG-(l,r)-Loop with a left identity $e_{\alpha}$ for each $\alpha \in Y$, and $S_{\alpha} \cap S_{\beta}=\varnothing$, $\alpha, \beta \in Y$ and $\alpha \neq \beta$. If, for all $a_{\alpha} \in S_{\alpha}$, for all $b_{\beta} \in S_{\beta}$, $a_{\alpha} * b_{\beta}=a_{\alpha} * e_{\alpha}$, and $b_{\beta} * a_{\alpha}=a_{\alpha}$, then $G$ is a strong $A G$ $(l, r)$-Loop. 
TABLE 6: Table of Example 6.

\begin{tabular}{lllllllll}
\hline$*$ & 1 & 2 & 3 & 4 & 5 & 6 & 7 & 8 \\
\hline 1 & 1 & 2 & 3 & 4 & 1 & 1 & 1 & 1 \\
2 & 2 & 1 & 4 & 3 & 2 & 2 & 2 & 2 \\
3 & 4 & 3 & 2 & 1 & 4 & 4 & 4 & 4 \\
4 & 3 & 4 & 1 & 2 & 3 & 3 & 3 & 3 \\
5 & 1 & 2 & 3 & 4 & 5 & 6 & 7 & 8 \\
6 & 1 & 2 & 3 & 4 & 6 & 5 & 8 & 7 \\
7 & 1 & 2 & 3 & 4 & 8 & 7 & 6 & 5 \\
8 & 1 & 2 & 3 & 4 & 7 & 8 & 5 & 6 \\
\hline
\end{tabular}

Proof. Suppose $G=\cup_{\alpha \in Y} S_{\alpha}$ is the groupoid, $Y$ is an AGband, for each $\alpha \in Y$, and $S_{\alpha}$ is a strong AG-(l,r)-Loop with a left identity $e_{\alpha}$ and $S_{\alpha} \cap S_{\beta}=\varnothing$ if $\alpha \neq \beta$ in $Y$.

We first prove that $G$ is an AG-groupoid. Let $a_{\alpha} \in S_{\alpha}$, $b_{\beta} \in S_{\beta}$, and $c_{\gamma} \in S_{\gamma}$ be arbitrary elements. Since $S_{\alpha}, S_{\beta}$, and $S_{\gamma}$ are strong AG- $(l, r)$-Loops, we have

$$
\begin{aligned}
\left(a_{\alpha} * b_{\beta}\right) * c_{\gamma} & =\left(a_{\alpha} * e_{\alpha}\right) * c_{\gamma} \\
& =\left(a_{\alpha} * e_{\alpha}\right) * e_{\alpha} \\
& =\left(e_{\alpha} * e_{\alpha}\right) * a_{\alpha} \quad \text { (by the left invertive law) } \\
& =e_{\alpha} * a_{\alpha}=a_{\alpha},
\end{aligned}
$$

where $\left(c_{\gamma} * b_{\beta}\right) * a_{\alpha}=b_{\beta} * a_{\alpha}=a_{\alpha}=\left(a_{\alpha} * b_{\beta}\right) * c_{\gamma}$. Since $S_{\alpha}$ is a strong AG- $(l, r)$-Loop, the left invertive law holds directly for elements $a_{\alpha}, b_{\alpha}, c_{\alpha} \in S_{\alpha}$. Thus, $G$ is an AG-groupoid.

For any $b_{\beta} \in S_{\beta}$, we have $n l r\left(b_{\beta}\right)=e_{\beta}$ and $\operatorname{olr}\left(b_{\beta}\right)$ $* b_{\beta}=b_{\beta} * \operatorname{olr}\left(b_{\beta}\right)=e_{\beta}$. Let $x \in G-S_{\beta}$, we denote $e_{x}$ is the left identity in $[x]_{\approx}, L S_{\beta}=\left\{x \mid x * b_{\beta}=x * e_{x}, b_{\beta} * x=x\right.$, $\left.x \in G-S_{\beta}\right\}$, and $R S_{\beta}=\left\{x \mid x * b_{\beta}=b_{\beta}, b_{\beta} * x=b_{\beta} * e_{\beta}, x \in\right.$ $G-S_{\beta}$. . Being $S_{\alpha} \cap S_{\beta}=\varnothing$ if $\alpha \neq \beta$ in $Y$, we can get $L S_{\beta} \cap S_{\beta} \cap R S_{\beta}=\varnothing$ and $L S_{\beta} \cup S_{\beta} \cup R S_{\beta}=G$.

Depending on $S_{\beta}$, we have three cases to discuss.

case 1. $L S_{\beta}=G-S_{\beta}, R S_{\beta}=\varnothing, x \in L S_{\beta}, x * b_{\beta}=x * e_{x}$, and $b_{\beta} * x=x$. Being $S_{\alpha} \cap S_{\beta}=\varnothing$ if $\alpha \neq \beta$ in $Y$, we can get $x * e_{x} \in[x]_{\approx}, x * b_{\beta} \notin S_{\beta}$. That is, there is no element $x \notin S_{\beta}$ such that $x * b_{\beta}=b_{\beta}$.

case 2. $L S_{\beta}=\varnothing, R S_{\beta}=G-S_{\beta}, x \in R S_{\beta}, x * b_{\beta}=b_{\beta}$, and $b_{\beta}$ $* x=b_{\beta} * e_{\beta}$. Being $S_{\alpha} \cap S_{\beta}=\varnothing$ if $\alpha \neq \beta$ in $Y$, we can get $b_{\beta} * x=b_{\beta} * e_{\beta} \in S_{\beta}$. That is, there is no element $x \notin S_{\beta}$ such that $x * b_{\beta}=b_{\beta}$ and $b_{\beta} * y=x$, and there exists $y \in G-S_{\beta}$.

case 3. $L S_{\beta} \neq \varnothing$ and $R S_{\beta} \neq \varnothing$, when $x \in L S_{\beta}, x * b_{\beta}=x *$ $e_{x} \notin S_{\beta}$, and $b_{\beta} * x=x \notin R S_{\beta}$; when $x \in R S_{\beta}, x * b_{\beta}=b_{\beta}$, $b_{\beta} * x=b_{\beta} * e_{\beta} \notin R S_{\beta}$. That is, there is no element $x \notin S_{\beta}$ such that $x * b_{\beta}=b_{\beta}$ and $b_{\beta} * y=x$, and there exists $y \in G-S_{\beta}$.

From all the above cases, $b_{\beta}$ has a unique $n l r\left(b_{\beta}\right)=e_{\beta}$ and $\left\{\operatorname{olr}\left(b_{\beta}\right)\right\} \subseteq S_{\beta}$. Consequently, $G$ is a strong AG- $(l, r)$ Loop.

Example 7. Let $G=\{1,2,3,4,5,6,7,8,9,10,11,12,13\}$, and the definition of operation $*$ on $G$ is shown in Table 7. An
TABLE 7: Table of Example 7.

\begin{tabular}{cccccccccccccc}
\hline$*$ & 1 & 2 & 3 & 4 & 5 & 6 & 7 & 8 & 9 & 10 & 11 & 12 & 13 \\
\hline 1 & 1 & 2 & 3 & 4 & 1 & 1 & 1 & 1 & 1 & 1 & 1 & 1 & 1 \\
2 & 2 & 1 & 4 & 3 & 2 & 2 & 2 & 2 & 2 & 2 & 2 & 2 & 2 \\
3 & 4 & 3 & 2 & 1 & 4 & 4 & 4 & 4 & 4 & 4 & 4 & 4 & 4 \\
4 & 3 & 4 & 1 & 2 & 3 & 3 & 3 & 3 & 3 & 3 & 3 & 3 & 3 \\
5 & 1 & 2 & 3 & 4 & 5 & 6 & 7 & 8 & 5 & 5 & 5 & 5 & 5 \\
6 & 1 & 2 & 3 & 4 & 6 & 5 & 8 & 7 & 6 & 6 & 6 & 6 & 6 \\
7 & 1 & 2 & 3 & 4 & 8 & 7 & 5 & 6 & 8 & 8 & 8 & 8 & 8 \\
8 & 1 & 2 & 3 & 4 & 7 & 8 & 6 & 5 & 7 & 7 & 7 & 7 & 7 \\
9 & 1 & 2 & 3 & 4 & 5 & 6 & 7 & 8 & 9 & 10 & 11 & 12 & 13 \\
10 & 1 & 2 & 3 & 4 & 5 & 6 & 7 & 8 & 10 & 11 & 12 & 13 & 9 \\
11 & 1 & 2 & 3 & 4 & 5 & 6 & 7 & 8 & 11 & 12 & 13 & 9 & 10 \\
12 & 1 & 2 & 3 & 4 & 5 & 6 & 7 & 8 & 12 & 13 & 9 & 10 & 11 \\
13 & 1 & 2 & 3 & 4 & 5 & 6 & 7 & 8 & 13 & 9 & 10 & 11 & 12 \\
\hline
\end{tabular}

AG-band $Y=\{1,5,9\} \quad$ and $\quad S_{1}=\{1,2,3,4\}, e_{1}=1$, $S_{5}=\{5,6,7,8\}, e_{5}=5$, and $S_{9}=\{9,10,11,12,13\}, e_{9}=9$. For any $a_{1} \in S_{1}, b_{5} \in S_{5}$, and $c_{9} \in S_{9}$, without losing generality, let $a_{1}=3, b_{5}=7$, and $c_{9}=10$, and we have $3 * 7=$ $3 * 1$ and $7 * 3=3,3 * 10=3 * 1$ and $10 * 3=3,7 * 10=$ $7 * 5$ and $10 * 7=7$, and $(3 * 7) * 10=(10 * 7) * 3$. The other cases can be verified; thus, $G$ is an AG-groupoid.

Let $c_{9}=10, L S_{9}=G-S_{9}=\{1,2,3,4,5,6,7,8\}$ and $R S_{9}$ $=\varnothing$; for all $x \in L S_{9}$, there is no element $x$ such that $x * 10=10$. That is, the element " 10 " has a unique $n l r(10)=$ 9 and $\{\operatorname{olr}(10)\}=\{13\} \subseteq S_{9}$.

Let $a_{1}=3, L S_{1}=\varnothing, R S_{1}=G-S_{1}=\{5,6,7,8,9,10,11,12,13\}$; for all $x \in R S_{1}, 3 * x=3 * e_{1}=3 * 1=4 \notin R S_{1}$; thus, there is no element $x$ such that there exists $y \in R S_{1}, x * 3=3,3 * y=x$. That is, the element " 3 " has a unique $n l r(3)=1$ and $\{\operatorname{olr}(3)\}=\{4\} \subseteq S_{1}$.

Let $b_{5}=7, L S_{5}=\{1,2,3,4\}$, and $R S_{5}=\{9,10,11,12,13\}$, when $x \in L S_{5}, x * 7=x * e_{x} \notin S_{5}, 7 * x=x \notin R S_{5}$; when $x \in R S_{5}, x * 7=7,7 * x=7 * e_{5}=7 * 5=8 \notin R S_{5}$. That is, there is no element $x \notin S_{5}$ such that $x * 7=7,7 * y=x$, and there exists $y \in G-S_{5}$. The element " 7 " has a unique $n \operatorname{lr}(7)=5$ and $\{\operatorname{olr}(7)\}=\{7\} \subseteq S_{5}$.

The other cases can be verified; thus, $G$ is a strong AG$(l, r)$-Loop.

Theorem 6. Let $(G, *)$ be a groupoid, $Y$ be an $A G$-band, $Y \subset G . G=\cup_{\alpha \in Y} S_{\alpha},\left(S_{\alpha}, *\right)$ be a strong AG-(l,r)-Loop with a left identity $e_{\alpha}$ for each $\alpha \in Y$, and $S_{\alpha} \cap S_{\beta}=\varnothing$, $\alpha, \beta \in Y, \alpha \neq \beta$. If, for all $a_{\alpha} \in S_{\alpha}$, for all $b_{\beta} \in S_{\beta}$, $a_{\alpha} * b_{\beta}=b_{\beta}, b_{\beta} * a_{\alpha}=b_{\beta} * e_{\beta}$, then $G$ is a strong $A G-(l, r)$ Loop.

Proof. Theorem 6 is proved similarly to Theorem 5 .

The strong AG-(l,r)-Loop constructed by Theorem 5 is not isomorphic to the strong AG- $(l, r)$-Loop constructed by Theorem 6.

Definition 12 (see [20]). An AG-(l,l)-Loop $(G, *)$ is called a strong AG-(l,l)-Loop if for all $a \in G, \operatorname{nll}(a)^{2}=n l l(a)$.

Example 8 illustrates that an AG- $(l, l)$-Loop is not always a strong AG-(l,l)-Loop. 
Example 8. Let $G=\{1,2,3,4,5,6,7,8\}$, and the definition of operation $*$ on $G$ is shown in Table 8 . From Definitions 5 and 7, G is both an AG-(l,l)-Loop and an AG-(l,r)-Loop. However, $n l l(1)=n l r(1)=3,3 * 3=4 \neq 3$; thus, it is neither a strong AG-(l,l)-Loop nor a strong AG-(l,r)-Loop.

Theorem 7. Let $(G, *)$ be an AG-groupoid. Then, the following three statements are equivalent:

(1) $G$ is a strong $A G-(l, r)-L o o p$

(2) $G$ is a strong $A G-(l, l)-L o o p$

(3) $G$ is an $A G-(l, l r)-L o o p$

Proof

$(1) \Longrightarrow(2)$. Suppose $G$ is a strong AG-(l,r)-Loop; from Definition 11, for all $a \in G$, there exist $n l r(a), \operatorname{olr}(a) \in G, \quad n l r(a) * a=a, \quad a * \operatorname{olr}(a)=n l r$ (a), and $n l r(a)^{2}=n \operatorname{lr}(a)$. Let $d=n \operatorname{lr}(a) * \operatorname{olr}(a)$, and we have $d * a=(n l r(a) * \operatorname{olr}(a)) * a=(a * o l r$ $(a)) * n l r \quad(a)=n l r(a)^{2}=n l r(a)$. From Definition $12, G$ is a strong AG-(l,l)-Loop.

$(2) \Longrightarrow(3)$. Suppose $G$ is a strong AG- $(l, l)$-Loop; from Definition 12, for all $a \in G$, there exist $\operatorname{nll}(a), \operatorname{oll}(a) \in G, \operatorname{nll}(a) * a=a, \operatorname{oll}(a) * a=\operatorname{nll}(a)$, and $\operatorname{nll}(a)^{2}=\operatorname{nll}(a)$. So, $a * \operatorname{oll}(a)=(\operatorname{nll}(a) * a)$ * oll $(a)=(\operatorname{oll}(a) * a) * \operatorname{nll}(a)=\operatorname{nll}(a)^{2}=\operatorname{nll}(a)$. By Definition 9, $G$ is an AG-(l,lr)-Loop.

$(3) \Longrightarrow(1)$. If $G$ is an AG-(l,lr)-Loop, from Definition 9, for all $a \in G$, there exist $\operatorname{nlr}(a), \operatorname{olr}(a) \in G$, $n \operatorname{lr}(a) * a=a$, and $\operatorname{olr}(a) * a=a * \operatorname{olr}(a)=n \operatorname{lr}(a)$. So, $n l r(a) * n l r(a)=(\operatorname{olr}(a) * a) * n l r(a)=(n l r(a)$ $* a) * \operatorname{olr}(a)=a * \operatorname{olr}(a)=n l r(a)$. By Definition $11, G$ is a strong AG- $(l, r)$-Loop.

Figure 1 shows the relationships among AG-(l,l)-Loop and AG- $(l, r)$-Loop. Here, A stands for AG-NET-Loop, B stands for strong AG-(l,r)-Loop shown in Example 4 rather than AG-NET-Loop, C stands for AG- $(l, r)$-Loop and AG$(l, l)$-Loop shown in Example 8, which is, however, not strong AG-(l,r)-Loop, D stands for AG- $(l, l)$-Loop rather than AG$(l, r)$-Loop, E stands for AG- $(l, r)$-Loop shown in Example 2 rather than AG- $(l, l)$-Loop, and $\mathrm{F}$ stands for AG-groupoid shown in Example 1, which is, however, not either AG- $(l, l)$ Loop or AG- $(l, r)$-Loop. A + B stands for strong AG- $(l, r)$ Loop, A+B + C + D stands for AG- $(l, l)$-Loop, A+B + C + E stands for $A G-(l, r)$-Loop, and $A+B+C+D+E+F$ stands for AG-groupoid.

\section{AG- $(r, r)$-Loop and AG- $(r, l)$-Loop}

Theorem 8. Let $(G, *)$ be an $A G-(r, r)$-Loop. Then,

(1) $G$ is an $A G-(r, l)-L o o p$

(2) $G$ is an $A G-(l, l)-L o o p$
TABLE 8: Table of Example 8.

\begin{tabular}{lllllllll}
\hline$*$ & 1 & 2 & 3 & 4 & 5 & 6 & 7 & 8 \\
\hline 1 & 2 & 4 & 3 & 1 & 7 & 5 & 6 & 8 \\
2 & 3 & 1 & 2 & 4 & 6 & 8 & 7 & 5 \\
3 & 1 & 3 & 4 & 2 & 8 & 6 & 5 & 7 \\
4 & 4 & 2 & 1 & 3 & 5 & 7 & 8 & 6 \\
5 & 8 & 6 & 5 & 7 & 6 & 8 & 7 & 5 \\
6 & 5 & 7 & 8 & 6 & 7 & 5 & 6 & 8 \\
7 & 7 & 5 & 6 & 8 & 5 & 7 & 8 & 6 \\
8 & 6 & 8 & 7 & 5 & 8 & 6 & 5 & 7 \\
\hline
\end{tabular}

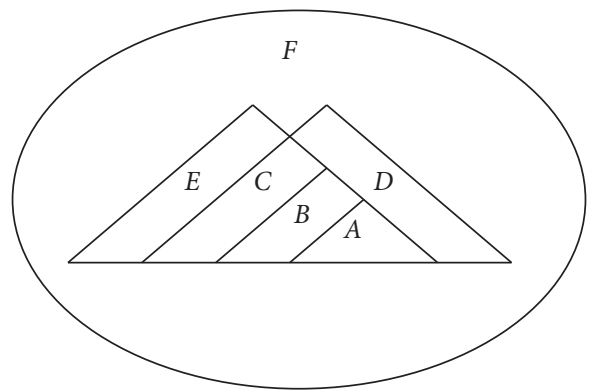

Figure 1: The relationships among AG-(l,l)-Loop and AG- $(l, r)$ Loop.

Proof

(1) Suppose $G$ is an AG-(r,r)-Loop; from Definition 6, for all $a \in G$, there exist $\operatorname{nrr}(a), \operatorname{orr}(a) \in G$, $a * n r r(a)=a$, and $a * \operatorname{orr}(a)=\operatorname{nrr}(a)$. Let $q=\operatorname{orr}(a) * \operatorname{nrr}(a)$, and we have $q * a=(\operatorname{orr}(a)$ $* n r r(a)) * a=(a * n r r(a)) \quad * \operatorname{orr}(a)=a * \operatorname{orr}($ $a)=\operatorname{nrr}(a)$. By Definition 8, G is an AG- $(r, l)$-Loop.

(2) Suppose $G$ is an AG-(r,r)-Loop; from Definition 6, for all $a \in G$, there exist $\operatorname{nrr}(a) \operatorname{orr}(a) \in G$, $a * \operatorname{nrr}(a)=a$, and $a * \operatorname{orr}(a)=\operatorname{nrr}(a)$. Let $d=\operatorname{nrr}(a)^{2}$ and $q=\operatorname{nrr}(a) * \operatorname{orr}(a)$, and we have $d * a=(n r r(a) * n r r(a)) * a=(a * n r r(a)) * n r r(a)$ $=a * \operatorname{nrr}(a)=a$ and $q * a=(\operatorname{nrr}(a) * \operatorname{orr}(a)) * a=$ $(a * \operatorname{orr}(a)) * \operatorname{nrr}(a)=\operatorname{nrr}(a) * n r r(a)=d$.

By Definition $5, G$ is an AG- $(l, l)$-Loop.

Definition 13. An AG- $(r, r)$-Loop $(G, *)$ is called a strong AG- $(r, r)$-Loop if for all $a \in G, \operatorname{nrr}(a)^{2}=\operatorname{nrr}(a)$.

Definition 14. An AG- $(r, l)$-Loop $(G, *)$ is called a strong AG- $(r, l)$-Loop if for all $a \in G, \operatorname{nrl}(a)^{2}=n r l(a)$.

Example 9 illustrates that an AG- $(r, r)$-Loop is not always a strong AG- $(r, r)$-Loop and an AG- $(r, l)$-Loop is not always a strong AG-(r,l)-Loop.

Example 9. Let $G=\{1,2,3,4,5,6,7,8\}$, and the definition of operation $*$ on $G$ is shown in Table 9. From Definitions 6, 8, 5, and 7, G is both an AG-(r,r)-Loop and an AG-(r,l)-Loop and an AG-(1,1)-Loop and AG-(1,r)-Loop. However, $n \operatorname{nr}(1)=4, \operatorname{nrl}(1)=4,4 * 4=3 \neq 4 ; \operatorname{nll}(1)=3, \operatorname{nlr}(1)=3,3$ $* 3=4 \neq 3$. Thus, $G$ is neither a strong AG-(r,r)-Loop nor a 
TABLE 9: Table of Example 9.

\begin{tabular}{lllllllll}
\hline$*$ & 1 & 2 & 3 & 4 & 5 & 6 & 7 & 8 \\
\hline 1 & 2 & 4 & 3 & 1 & 3 & 1 & 2 & 4 \\
2 & 3 & 1 & 2 & 4 & 2 & 4 & 3 & 1 \\
3 & 1 & 3 & 4 & 2 & 4 & 2 & 1 & 3 \\
4 & 4 & 2 & 1 & 3 & 1 & 3 & 4 & 2 \\
5 & 1 & 3 & 4 & 2 & 6 & 8 & 7 & 5 \\
6 & 4 & 2 & 1 & 3 & 7 & 5 & 6 & 8 \\
7 & 2 & 4 & 3 & 1 & 5 & 7 & 8 & 6 \\
8 & 3 & 1 & 2 & 4 & 8 & 6 & 5 & 7 \\
\hline
\end{tabular}

strong AG-(r,l)-Loop nor a strong AG-(l,l)-Loop nor a strong AG-(l,r)-Loop.

Theorem 9. Let $(G, *)$ be an AG-groupoid. Then, the following three statements are equivalent:

(1) $G$ is a strong $A G-(r, r)-L o o p$

(2) $G$ is a strong $A G-(r, l)-L o o p$

(3) $G$ is an AG-NET-Loop

Proof

$(1) \Longrightarrow(2)$. Suppose $G$ is a strong AG- $(r, r)$-Loop; from Definition 13, for all $a \in G$, there exist $\operatorname{nrr}(a)$, $\operatorname{orr}(a) \in G, a * \operatorname{nrr}(a)=a, a * \operatorname{orr}(a)=\operatorname{nrr}(a)$, and $\operatorname{nrr}(a)^{2}=\operatorname{nrr}(a)$. Let $q=\operatorname{orr}(a) * \operatorname{nrr}(a)$, and we have $\quad q * a=(\operatorname{orr}(a) * n r r(a)) * a=(a * n r r(a))$ * orr $(a)=a * \operatorname{orr}(a)=\operatorname{nrr}(a)$. By Definition 14, $G$ is a strong AG- $(r, l)$-Loop.

$(2) \Longrightarrow(3)$. Suppose $G$ is a strong AG- $(r, l)$-Loop; from Definition 14, for all $a \in G$, there exist $\operatorname{nrl}(a), \operatorname{orl}(a) \in G, \quad a * \operatorname{nrl}(a)=a$, $\operatorname{orl}(a) * a=\operatorname{nrl}(a)$, and $\operatorname{nrl}(a)^{2}=\operatorname{nrl}(a)$. So, $\operatorname{nrl}(a) * a=(\operatorname{nrl}(a) * n r l$

$(a)) * a=(a * n r l(a)) * n r l(a)=a * n r l(a)=a$ and $a * \operatorname{orl}(a)=(\operatorname{nrl}(a) * a) * \operatorname{orl}(a)=(\operatorname{orl}(a) * a) * n$ $r l(a)=n r l(a)^{2}=n r l(a)$. By Definition 3, $G$ is an AG-NET-Loop.

$(3) \Longrightarrow(1)$. It is obvious that an AG-NET-Loop is a strong AG- $(r, r)$-Loop.

Figure 2 shows the relationships among AG-( $r, l)$-Loop and AG- $(l, r)$-Loop. Here, A stands for AG-NET-Loop, B stands for AG- $(r, l)$-Loop and strong AG- $(l, r)$-Loop shown in Example 4, which is, however, not AG-NET-Loop, C stands for AG- $(r, l)$-Loop and AG- $(l, r)$-Loop shown in Example 9, which is, however, not strong AG- $(l, r)$-Loop, D stands for AG- $(r, l)$-Loop rather than AG- $(l, r)$-Loop, E stands for strong AG-(l,r)-Loop rather than AG- $(r, l)$-Loop, F stands for AG- $(l, r)$-Loop shown in Example 2, which is, however, not either AG- $(r, l)$-Loop or strong AG- $(l, r)$-Loop, and G stands for AG-groupoid shown in Example 1, which is, however, not either AG- $(l, r)$-Loop or AG- $(r, l)$-Loop. A + B + E stands for strong AG- $(l, r)$-Loop, $\mathrm{A}+\mathrm{B}+\mathrm{C}+\mathrm{D}$ stands for AG- $(r, l)$ Loop, $\mathrm{A}+\mathrm{B}+\mathrm{C}+\mathrm{E}+\mathrm{F}$ stands for AG- $(l, r)$-Loop, and $\mathrm{A}+\mathrm{B}+\mathrm{C}+\mathrm{D}+\mathrm{E}+\mathrm{F}+\mathrm{G}$ stands for AG-groupoid.

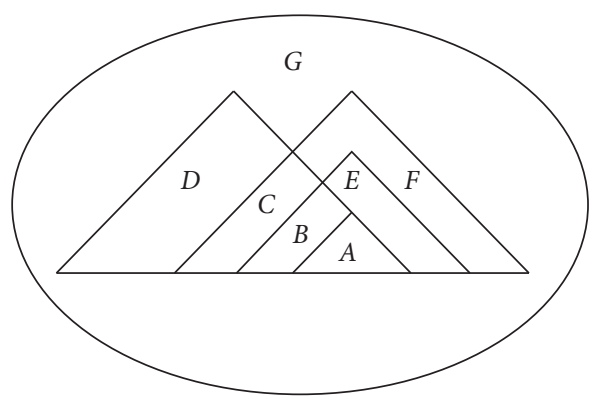

FIgure 2: The relationships among AG- $(r, l)$-Loop and AG- $(l, r)$ Loop.

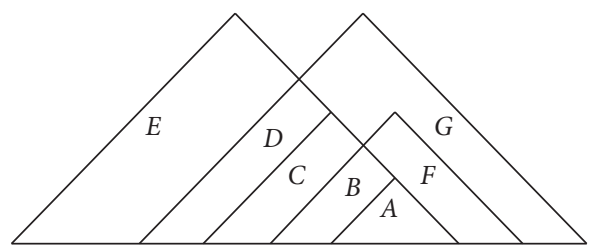

FIgUre 3: The relationships among AG- $(r, l)$-Loop and AG- $(l, l)-$ Loop.

Figure 3 shows the relationships among AG- $(r, l)$-Loop and AG-(l,l)-Loop. Here, A stands for AG-NET-Loop, B stands for AG- $(r, r)$-Loop and strong AG- $(l, l)$-Loop shown in Example 4, which is, however, not AG-NET-Loop, C stands for AG- $(r, r)$-Loop shown in Example 9 rather than strong AG- $(l, l)$-Loop, D stands for AG- $(r, l)$-Loop and AG- $(l, l)$ Loop rather than AG- $(r, r)$-Loop, E stands for AG- $(r, l)$-Loop rather than AG- $(l, l)$-Loop, F stands for strong AG- $(l, l)$-Loop rather than AG- $(r, l)$-Loop, and G stands for AG- $(l, l)$-Loop, which is, however, not either AG- $(r, l)$-Loop or a strong AG$(l, l)$-Loop. $\mathrm{A}+\mathrm{B}+\mathrm{C}$ stands for $\mathrm{AG}-(r, r)$-Loop, $\mathrm{A}+\mathrm{B}+\mathrm{F}$ stands for strong AG-(1,l)-Loop, A + B + C + D + E stands for $A G-(r, l)$-Loop, and $A+B+C+D+F+G$ stands for $A G-$ $(l, l)$-Loop.

\section{Alternative Quasi AG-NET-Loop}

Definition 15. Let $(G, *)$ be an AG-NET-Loop (AG-(1,1)Loop, AG-(l,r)-Loop, AG-(r,r)-Loop, and AG-(r,l)-Loop). Then, $G$ is called a right alternative AG-NET-Loop (AG-(1,1)Loop, AG-(l,r)-Loop, AG-(r,r)-Loop, and AG-(r,l)-Loop) if $b *(a * a)=(b * a) * a$, for all $a, b \in G$.

Definition 16. Let $(G, *)$ be an AG-NET-Loop (AG-(1,1)Loop, AG-(l,r)-Loop, AG-(r,r)-Loop, and AG-(r,l)-Loop). Then, $G$ is called an alternative AG-NET-Loop (AG-(l,l)Loop, AG-(l,r)-Loop, AG-(r,r)-Loop, and AG-(r,l)-Loop), if for all $a, b \in G,(a * a) * b=a *(a * b), a *(b * b)=(a *$ b) $* b$.

Example 10 illustrates that an AG-NET-Loop is not always an alternative AG-NET-Loop.

Example 10. Let $G=\{1,2,3,4,5,6,7\}$, and the definition of operation * on $G$ is shown in Table 10 . By Definition $3, G$ is 
TABle 10: Table of Example 10.

\begin{tabular}{llllllll}
\hline$*$ & 1 & 2 & 3 & 4 & 5 & 6 & 7 \\
\hline 1 & 1 & 4 & 2 & 3 & 3 & 1 & 2 \\
2 & 3 & 2 & 4 & 1 & 1 & 3 & 4 \\
3 & 4 & 1 & 3 & 2 & 2 & 4 & 3 \\
4 & 2 & 3 & 1 & 4 & 4 & 2 & 1 \\
5 & 2 & 3 & 1 & 4 & 5 & 2 & 1 \\
6 & 1 & 4 & 2 & 3 & 3 & 6 & 2 \\
7 & 4 & 1 & 3 & 2 & 2 & 4 & 7 \\
\hline
\end{tabular}

an AG-NET-Loop. However, $G$ is not an alternative AGNET-Loop because $(3 * 4) * 4 \neq 3 *(4 * 4)$.
Theorem 10. Let $(G, *)$ be an AG-NET-Loop. Then, the following three statements are equivalent:

(1) $G$ is a right alternative AG-NET-Loop

(2) $G$ is a commutative AG-NET-Loop

(3) $G$ is an alternative AG-NET-Loop

Proof

$(1) \Rightarrow(2)$. Suppose $G$ is a right alternative AG-NETLoop; from Definition 15, for all $a, b \in G$,

$$
\begin{aligned}
& \qquad \begin{aligned}
a * \operatorname{neut}(b) & =a *(\text { neut }(b) * \text { neut }(b)) \\
& =(a * \operatorname{neut}(b)) * \operatorname{neut}(b) \\
& =(\operatorname{neut}(b) * \operatorname{neut}(b)) * a \\
& =\operatorname{neut}(b) * a,
\end{aligned} \\
& \text { so } \\
& \begin{aligned}
& a * b=(\operatorname{neut}(a) * a) *(b * \operatorname{neut}(b)) \\
&=(\operatorname{neut}(a) * b) *(a * \operatorname{neut}(b))(\text { by the medial law }) \\
&=(b * \operatorname{neut}(a)) *(\operatorname{neut}(b) * a) \\
&=(b * \operatorname{neut}(b)) *(\operatorname{neut}(a) * a) \\
&=b * a .
\end{aligned}
\end{aligned}
$$$$
=(a * \text { neut }(b)) * \text { neut }(b)
$$

Consequently, $G$ is a commutative AG-NET-Loop.

(2) $\Rightarrow(3)$. If $G$ is a commutative AG-NET-Loop, for all $m, n \in G, \quad m *(n * n)=(n * n) * m=(m * n) * n$ and $\quad(m * m) * n=$ $(n * m) * m=m *(n * m)=m *(m * n)$. By Definition $16, G$ is an alternative AG-NET-Loop.

(3) $\Rightarrow(1)$. It is obvious that an alternative AG-NET-Loop is a right alternative AG-NET-Loop.

Theorem 11 (see [23]). Let $(G, *)$ be a locally associative $A G$-groupoid. If $G$ is finite, then there exists $a \in G, a^{2}=a$.

Theorem 12. Let $(G, *)$ be a right alternative $A G-(r, l)$ Loop. If $G$ is finite, then, for all $a \in G$, there exist $s, p \in G, a * s=a, p * a=s$, and $s^{2}=s$.

Proof. If $G$ is a finite right alternative AG- $(r, l)$-Loop. Then, for all $a \in G$, there exist $s, p \in G, a * s=a$, and $p * a=s$, and we have $a * s^{2}=a *(s * s)=(a * s) * s=a * s=a$.

When $k \in Z^{+}, k>2$, (by Theorem 1 (2))

(by the right alternative law)

(by the left invertive law)

$$
\begin{aligned}
a * s^{k} & =(a * s) *\left(s^{2} * s^{k-2}\right) \\
& =\left(a * s^{2}\right) *\left(s * s^{k-2}\right) \quad(\text { by the medial law }) \\
& =a * s^{k-1} \\
& =\cdots \ldots \\
& =a * s^{2}=a .
\end{aligned}
$$

Thus, $s, s^{2}, s^{3}, \ldots, s^{k}, \ldots$ are all right neutral element.

By Theorem 11, we get that there is an idempotent right neutral element in $G$.

Theorem 13 (see [23]). Let $(G, *)$ be a finite alternative $A G-(l, l)$-Loop. Then, $G$ is a strong AG-(l,l)-Loop.

Theorem 14. Let $(G, *)$ be an AG-groupoid. Then, the following three statements are equivalent:

(1) $G$ is a finite right alternative $A G-(r, l)$-Loop

(2) $G$ is a finite alternative AG-NET-Loop

(3) $G$ is a finite alternative $A G-(l, l)-L o o p$

Proof

$(1) \Rightarrow(2)$. If $G$ is a finite right alternative AG- $(r, l)$-Loop, applying Theorem 12 , we get that $G$ is a strong AG$(r, l)$-Loop. From Theorem 9, we get that $G$ is a right alternative AG-NET-Loop. Applying Theorem 10, G is a finite alternative AG-NET-Loop.

$(2) \Rightarrow(3)$. It is obvious that a finite alternative AG-NETLoop is a finite alternative AG- $(l, l)$-Loop.

(3) $\Rightarrow(1)$. If $G$ is a finite alternative AG-(l,l)-Loop, applying Theorem 13, we get that $G$ is a strong AG-(1,1)Loop. From Definition 12, for all $a \in G$, there exist $\operatorname{nll}(a), \operatorname{oll}(a) \in G, \operatorname{nll}(a) * a=a, \operatorname{oll}(a) * a=\operatorname{nll}(a)$, and $\operatorname{nll}(a)^{2}=\operatorname{nll}(a)$. We have 
TABLE 11: Table of Example 11.

\begin{tabular}{|c|c|c|c|c|c|c|c|}
\hline * & 1 & 2 & 3 & 4 & 5 & 6 & 7 \\
\hline 1 & 2 & 5 & 4 & 1 & 3 & 1 & 1 \\
\hline 2 & 5 & 3 & 1 & 2 & 4 & 2 & 2 \\
\hline 3 & 4 & 1 & 5 & 3 & 2 & 3 & 3 \\
\hline 4 & 1 & 2 & 3 & 4 & 5 & 4 & 4 \\
\hline 5 & 3 & 4 & 2 & 5 & 1 & 5 & 5 \\
\hline 6 & 1 & 2 & 3 & 4 & 5 & 6 & 4 \\
\hline 7 & 1 & 2 & 3 & 4 & 5 & 4 & 7 \\
\hline
\end{tabular}

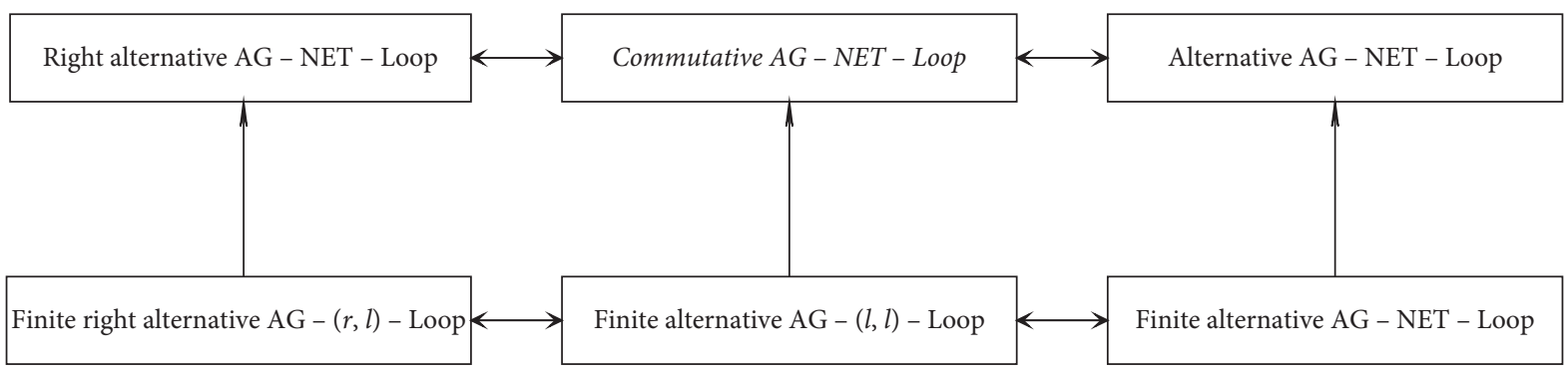

FIGURE 4: The relationships among alternative AG-NET-Loop and other alternative quasi AG-NET-Loops.

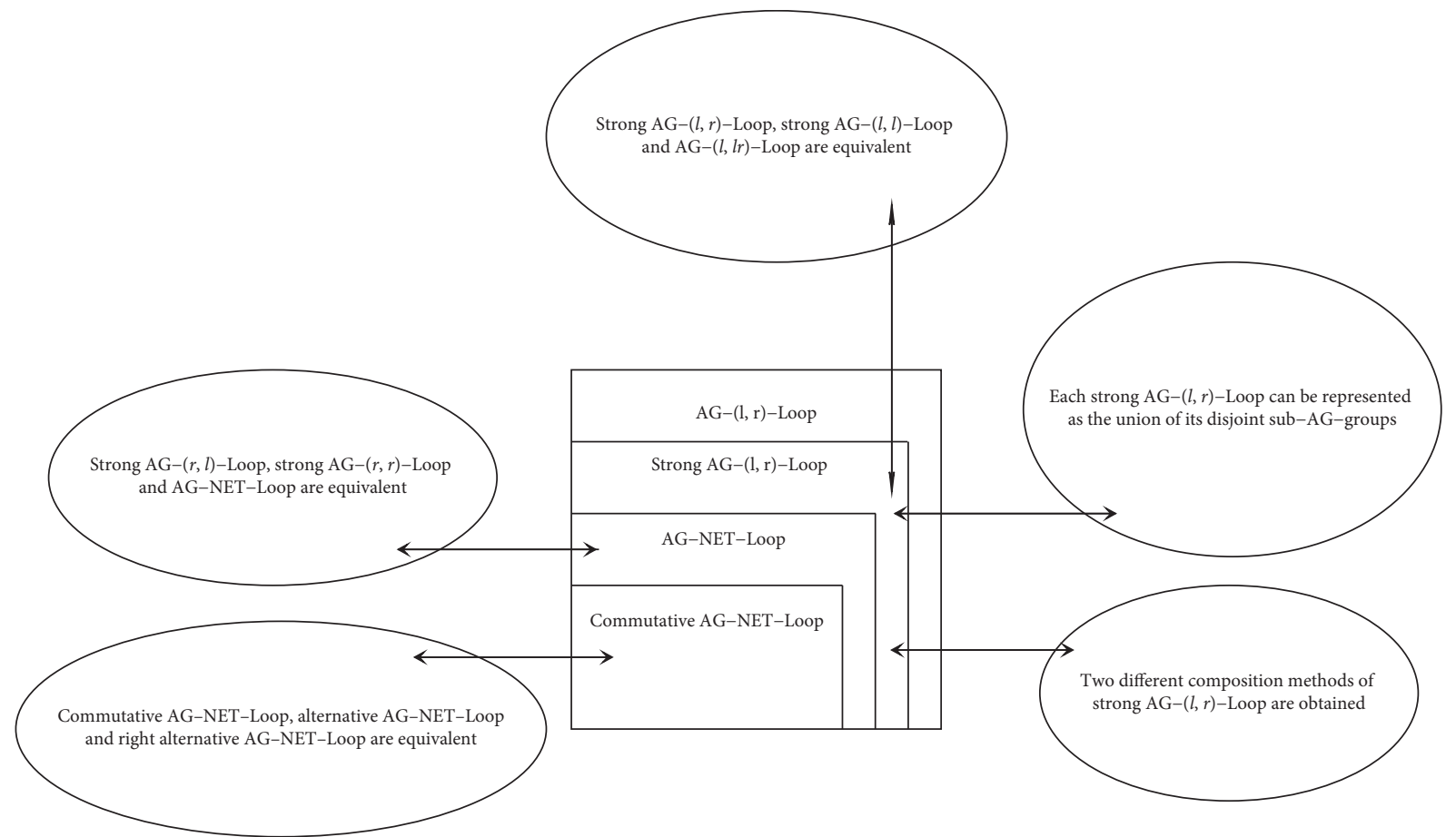

Figure 5: The main results of this paper.

$$
\begin{array}{rlrl}
a * \operatorname{nll}(a) & =a *(\operatorname{nll}(a) * \operatorname{nll}(a)) & \\
& =(a * \operatorname{nll}(a)) * \operatorname{nll}(a) & & \text { (by the right alternative law) } \\
& =(\operatorname{nll}(a) * \operatorname{nll}(a)) * a & & \text { (by the left invertive law }) \\
& =\operatorname{nll}(a) * a=a .
\end{array}
$$

By Definition 15, $G$ is a finite right alternative AG- $(r, l)$ Loop.
Example 11. Let $G=\{1,2,3,4,5,6,7\}$, and the definition of operation * on $G$ is shown in Table 11 . We can easily verify that $G$ satisfies the alternative law. Being each element in $G$ has a neutral element and an opposite element; by Definition $16, G$ is a finite alternative AG-NET-Loop. Obviously, a finite alternative AG-NET-Loop is both a finite right alternative AG-(r,l)-Loop and a finite alternative AG-(l,1)Loop. Since for all $a, b \in G$ and $a * b=b * a$, we have $G$ as a commutative AG-NET-Loop.

Figure 4 shows the relationships among alternative AGNET-Loop and other alternative quasi AG-NET-Loops. In 
Figure 4, we prove that the right alternative AG-NET-Loop is equivalent to the commutative AG-NET-Loop, and the commutative AG-NET-Loop is equivalent to the alternative AG-NET-Loop. As the finite right alternative AG- $(r, l)$-Loop is equivalent to the finite alternative AG-(l,l)-Loop, the finite alternative AG-(l,l)-Loop is equivalent to the finite alternative AG-NET-Loop; therefore, they are equivalent to each other.

\section{Conclusion}

In this paper, the AG- $(l, r)$-Loop and AG- $(r, l)$-Loop have been introduced, the structure of the quasi AG-NET-Loops have been studied further, and some important results have been obtained. We prove that the strong AG- $(l, r)$-Loop, the strong AG-(l,l)-Loop, and the AG- $(l, l r)$-Loop are equivalent (see Theorem 7 ); the strong AG- $(r, l)$-Loop, the strong AG$(r, r)$-Loop, and the AG-NET-Loop are equivalent (see Theorem 9); the commutative AG-NET-Loop, the alternative AG-NET-Loop, and the right alternative AG-NET-Loop are equivalent (see Theorem 10). Furthermore, the decomposition theorem of strong AG- $(l, r)$-Loop (see Theorem 4 ) and two different ways how to make a strong AG- $(l, r)$ Loop are obtained (see Theorem 5 and Theorem 6), thus illuminating the structure of strong AG-(l,r)-Loop. Figure 5 shows the main results of this paper. Future efforts will be directed towards discussing the relationship between strong AG- $(l, r)$-Loop and other related AG-groupoid bands, such as root of band, AG-4-band, and AG-3-band (see [24]).

\section{Data Availability}

No data were used to support this study.

\section{Conflicts of Interest}

The authors declare that there are no conflicts of interest regarding the publication of this paper.

\section{Acknowledgments}

This research was supported by Scientific Research Program funded by Shaanxi Provincial Education Department (Program No. 20JK0549).

\section{References}

[1] M. Kazim and M. Naseeruddin, "On almost semigroups," The Aligarh Bulletin of Mathematics, vol. 2, pp. 1-7, 1972.

[2] M. Khan, Faisal, and V. Amjid, "On some classes of AbelGrassmann's groupoids," Journal of Advanced Research in Pure Mathematics, vol. 3, pp. 109-119, 2011.

[3] Faisal, A. Khan, and B. Davvaz, "On fully regular AGgroupoids," Afrika Matematika, vol. 25, no. 2, pp. 449-459, 2014.

[4] M. Rashad, I. Ahmad, M. Shah, and Z. U. A. Khuhro, "Left transitive AG-groupoids," Sindh University Research Journal (Science Series), vol. 46, pp. 547-552, 2014.

[5] M. Iqbal and I. Ahmad, "Ideals in CA-AG-groupoids," Indian Journal of Pure and Applied Mathematics, vol. 49, no. 2, pp. 265-284, 2018.
[6] F. Smarandache, "Neutrosophic set-a generalization of the intuitionistic fuzzy set," International Journal of Pure and Applied Mathematics, vol. 24, pp. 287-297, 2005.

[7] M. Abdel-Basset, A. Gamal, L. H. Son, and F. Smarandache, "A bipolar neutrosophic multi criteria decision making framework for professional selection," Applied Sciences, vol. 10, no. 4, p. 1202, 2020.

[8] K. Mishra, I. Kandasamy, V. Kandasamy, and F. Smarandache, "A novel framework using neutrosophy for integrated speech and text sentiment analysis," Symmetry, vol. 12 , no. 10 , p. $1715,2020$.

[9] J. Kavikumar, D. Nagarajan, S. P. Tiwari, S. Broumi, and F. Smarandache, "Composite neutrosophic finite automata," Neutrosophic Sets and Systems, vol. 36, pp. 282-291, 2020.

[10] E. Rashno, B. Minaei-Bidgoli, and Y. Guo, "An effective clustering method based on data indeterminacy in neutrosophic set domain," Engineering Applications of Artificial Intelligence, vol. 89, Article ID 103411, 2020.

[11] G. Cai, Y. Guo, W. Chen, H. Zeng, Y. Zhou, and Y. Lu, "Computer-aided detection and diagnosis of microcalcification clusters on full field digital mammograms based on deep learning method using neutrosophic boosting," Multimedia Tools and Applications, vol. 79, no. 23-24, pp. 17147-17167, 2020.

[12] X. Zhang, X. Mao, F. Smarandache, and C. Park, "On homomorphism theorem for perfect neutrosophic extended triplet groups," Information, vol. 9, no. 9, p. 237, 2018.

[13] X. Zhang, Y. Ma, and P. Yu, "On two conjectures of Abel Grassmann's groupoids," Symmetry, vol. 11, no. 6, p. 816, 2019.

[14] X. Zhang and X. Wu, "Involution Abel-Grassmann's groups and filter theory of Abel-Grassmann's groups," Symmetry, vol. 11, no. 4, p. 553, 2019.

[15] M. Abdel-Basset, V. Chang, M. Mohamed, and F. Smarandche, "A refined approach for forecasting based on neutrosophic time series," Symmetry, vol. 11, no. 4, p. 457, 2019.

[16] W. Yuan and X. Zhang, "Regular CA-groupoids and cyclic associative neutrosophic extended triplet groupoids (CANET-groupoids) with green relations," Mathematics, vol. 8, no. 2, p. 204, 2020.

[17] M. Hu, F. Smarandache, and X. Zhang, "On neutrosophic extended triplet LA-hypergroups and strong pure LA-semihypergroups," Symmetry, vol. 12, no. 1, p. 163, 2020.

[18] X. Zhang, X. Wu, X. Mao, F. Smarandache, and C. Park, "On neutrosophic extended triplet groups (loops) and AbelGrassmann's groupoids (AG-groupoids)," Journal of Intelligent \& Fuzzy Systems, vol. 37, no. 4, pp. 5743-5753, 2019.

[19] X. Zhang, X. Wu, F. Smarandache, and M. Hu, "Left (right)quasi neutrosophic triplet loops (groups) and generalized BEalgebras," Symmetry, vol. 10, no. 7, p. 241, 2018.

[20] X. Wu and X. Zhang, "The decomposition theorems of AGneutrosophic extended triplet loops and strong AG-(1,1)loops," Mathematics, vol. 7, p. 268, 2019.

[21] F. Smarandache, Neutrosophic Perspectives: Triplets, Duplets, Multisets, Hybrid Operators, Modal Logic, Hedge Algebras and Applications, Pons Publishing House, Brussels, Belgium, 2017.

[22] P. V. Protić, "Some remarks on Abel-Grassmann's groups," Quasigroups and Related Systems, vol. 20, pp. 267-274, 2012.

[23] X. An, X. Zhang, and Y. Ma, "Generalized Abel-Grassmann's neutrosophic extended triplet loop," Mathematics, vol. 7, no. 12, p. 1206, 2019.

[24] N. Stevanović and P. V. Protić, "Composition of AbelGrassmann's 3-bands," Novi Sad Journal of Mathematics, vol. 34, pp. 175-182, 2004. 